\title{
DEMO: Real-time Edge Analytics and Concept Drift Computation for Efficient Deep Learning From Spectrum Data
}

\author{
Zaheer Khan*, Janne Lehtomäki*, Adnan Shahid ${ }^{\dagger}$, and Ingrid Moerman ${ }^{\dagger}$ \\ University of Oulu*, Oulu, Finland, and University of Ghent*, Ghent, Belgium \\ zaheer.khan@oulu.fi
}

\begin{abstract}
Cloud managed wireless network resource configuration platforms are being developed for efficient network utilization. These platforms can improve their performance by utilizing real-time edge analytics of key wireless metrics, such as wireless channel utilization (CU). This paper demonstrates a real-time spectrum edge analytics system which utilizes fieldprogrammable gate array (FPGA) to process in real-time hundreds of millions of streaming inphase and quadrature (IQ) samples per second. It computes not only mean and maximum values of $\mathrm{CU}$ but also computes histograms to obtain probability distribution of $\mathrm{CU}$ values. It sends in real-time these descriptive statistics to an entity which collects these statistics and utilises them to train a deep learning model for prediction of future $\mathrm{CU}$ values. Even though utilization in a wireless channel can often exhibit stable seasonal patterns, they can be affected by uncertain usage events, such as sudden increase/decrease in channel usage within a certain time period. Such changes can unpredictably drift concept of $\mathrm{CU}$ data (underlying distribution of incoming $\mathrm{CU}$ data) over time. In general, concept drift can deteriorate the prediction performance of deep learning models which in turn can impact the performance of cloud managed resource allocation solution. This paper also demonstrates a real-time concept drift computation method which measures the changes in the probability distribution of $\mathrm{CU}$ data. Our implemented demonstration includes: 1) spectrum analytics and concept drift computation device which is realized in practical implementation by prototyping it on a low-cost ZedBoard with AD9361 RF transceiver attached to it. ZedBoard is equipped with a Xilinx Zynq-7000 system on chip; 2) a laptop which is connected to the Zedboard and it provides graphical real-time displays of computed $\mathrm{CU}$ values, $\mathrm{CU}$ histograms, and concept drift computation values. A laptop is also used to develop a deep learning based model for prediction of future $\mathrm{CU}$ values. For the INFOCOM we will show a live demonstration of the complete prototyped system in which the device performs realtime computations in an unlicensed frequency channel following the implemented algorithms on the FPGA of a Zedboard.
\end{abstract}

\section{BACKGROUND AND MOTIVATION}

Cloud managed networks are being deployed to ensure efficient and robust resource allocation for wireless connectivity [1]. Use of descriptive analytics to track the right metrics and deep learning to make realistic predictions will play an important role in efficient cloud managed network design. Wireless radio environments are generally dynamic and often require real-time processing of key metric data to gain realistic insights. Processing and analyzing key metric data at the edge, close to its point of generation, can achieve better realtime processing and can also reduce data transfer overhead to improve efficiency [2]. To this end, hardware accelerated dedicated spectrum edge analytics modules are being deployed

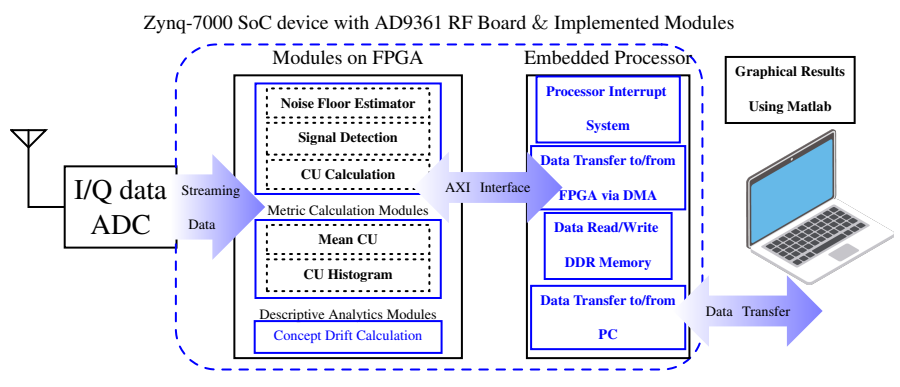

Fig. 1. Various components of our prototyped spectrum analytics system.

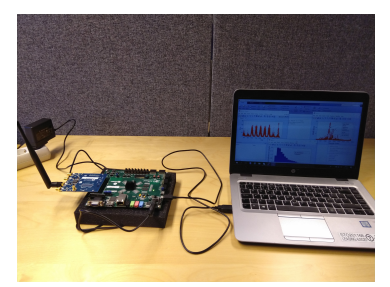

Fig. 2. Demo setup showing Zedboard (with AD9361 RF transceiver) performing spectrum analytics and Laptop displaying graphical results.

inside access points/base stations by wireless industry. For example, Meraki cloud managed network of Cisco utilizes dedicated radio module called Air Marshal in each Meraki AP [3]. The dedicated module in each access point (AP) monitors radio environment, processes data to obtain useful information, and sends this information to the cloud server.

This paper demonstrates a prototype of an edge spectrum analytics device on a Xilinx's Zynq-7000 SoC. The implemented device can process millions of in-phase and quadrature (IQ) samples to obtain in real-time descriptive statistics relating to a key wireless metric, such as wireless channel utilization $(\mathrm{CU})$ which is often given in a percentage between $0 \%$ to $100 \%$ and indicates the usage amount of a wireless channel within some measuring time interval $t$. Unlike existing approaches which tend to focus only on mean CU values [3], our implemented device computes not only mean CU values, but also maximum $\mathrm{CU}$ values, and histograms of $\mathrm{CU}$ values to obtain entire probability distribution. These CU statistics are sent to an entity which can use them to train a deep learning model for making realistic predictions about future $\mathrm{CU}$ values which in turn can be used for proactive resource allocation decisions. Our analytics device processes real spectrum data 


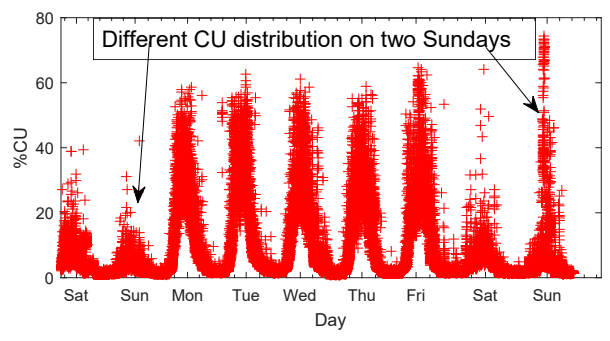

(a) Collected CU data over 9 Days.

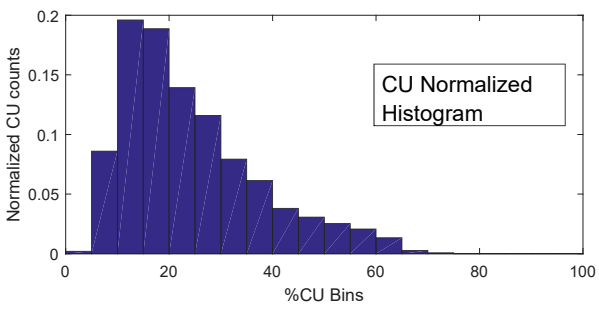

(c) Computed 20 bin CU histogram.

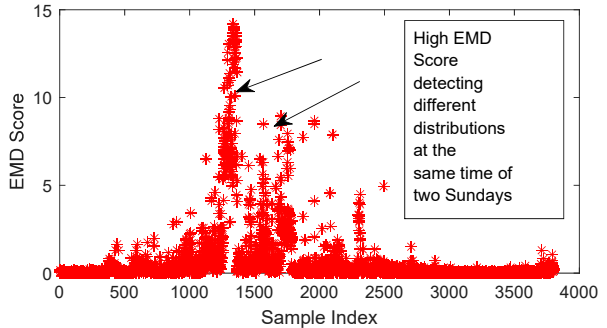

(b) Drift Detection on two Sundays using EMD.

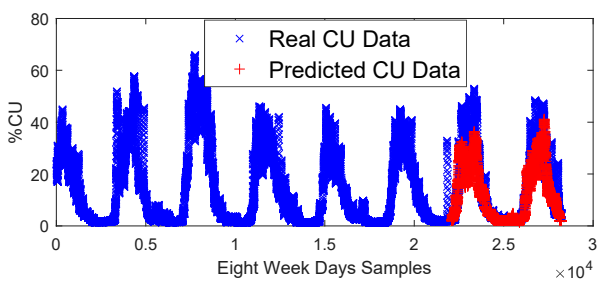

(d) Deep learning based predictions on CU data.

Fig. 3. Examples of $\mathrm{CU}$ analytics \& concept drift results using the prototyped device for computations over a period of two weeks in the University of Oulu.

which can change constantly in time. This can lead to degradation in predictive performance of deep learning model at the cloud which typically assumes a static distribution for input and output variables. This problem of changing underlying data distributions over time is called concept drift. To address this challenge, the implemented edge analytics device also performs real-time concept drift computation which measures the changes overtime in the probability distribution of $\mathrm{CU}$ data. Our drift computation is based on a statistical distance based method called earth mover's distance (EMD). In simple words, EMD can be explained as considering two probability distributions as piles of dirt and calculating the minimum amount of effort needed to reshape the first pile so that it has the same shape as the second pile. Two exactly matching $\mathrm{CU}$ probability distributions will have zero EMD, while the maximum value for the EMD is $B-1$, where $B$ is the number of bins of the CU histogram.

\section{Demonstration Overview}

In Fig. 1, we illustrate various components of the implemented spectrum analytics system: 1) An ADC of AD9361 (a high performance agile RF transceiver) for streaming reception of over-the-air IQ samples from multiple active wireless devices in a frequency channel; 2) Various modules implemented on the FPGA of a Zynq-7000 SoC device, such as noise floor estimation, signal detection, $\mathrm{CU}$ calculations, $\mathrm{CU}$ histogram and concept drift computations; 3) Implemented modules on the Embedded ARM processor of the Zynq SoC, such as sample transfer via direct memory access (DMA) to/from FPGA; and 4) a communication module between the implemented device and a laptop for streaming transfer of processed $\mathrm{CU}$ analytics. The figure also shows a laptop which is used to show real-time graphical output results, and is also used to perform deep learning based predictions on collected $\mathrm{CU}$ samples. Complete demo setup is shown in Fig. 2. Examples of results computed using the implemented device are shown in Fig. 3.
These results will be demonstrated graphically in real-time for the INFOCOM demo.

The CU statistics processing modules are implemented on the FPGA by incorporating new IP cores in the FMCOMMS2 HDL reference design of Analog Devices (see [4], and references there in). The FPGA modules are prototyped using three Xilinx tools: 1) Vivado High Level Synthesis; 2) System Generator for DSP; and 3) Vivado. For embedded processor programming Xilinx's SDK tool is utilized. Real-time graphical results are displayed using Matlab taking samples from the prototyped analytics device. Deep learning model is built on a laptop using Keras with Tensorflow backend.

\section{CONCLUding REMARKS}

Using real over-the-air IQ samples and Xilinx's Zynq$7000 \mathrm{SoC}$, we combine real spectrum data, hardware/software related tools to prototype a real-time spectrum analytics and concept drift computation device. The computed analytics can be exploited in a cloud managed wireless network in a variety of ways. For example, they can be used: 1) to develop a deep learning model for predictions; and 2) to detect concept drift which can allow to update/improve the developed deep learning model.

Video Link: http://www.ee.oulu.fi/ zaheer/infocomvideo/

\section{REFERENCES}

[1] J. Wang, J. Wang, Y. Wu, J. Wang, H. Zhu, M. Lin, and J. Wang, "A machine learning framework for resource allocation assisted by cloud computing," IEEE Network, vol. 32, no. 2, pp. 144-151, March 2018.

[2] Q. Zhang, W. Shi, and H. Zhong, "Firework: Data processing and sharing for hybrid cloud-edge analytics," IEEE Transactions on Parallel and Distributed Systems, vol. 29, no. 9, pp. 2004-2017, Sep. 2018.

[3] Cisco, "Meraki cloud controller," Meraki, Cisco Systems, Tech. Rep., March, 2017. [Online]. Available: \{https://documentation.meraki.com/Architectures_and_Best_Practices/ Cisco_Meraki_Best_Practice_Design/Meraki_Cloud_Architecture

[4] Z. Khan and J. J. Lehtomäki, "FPGA-assisted real-time RF wireless data analytics system: Design, implementation, and statistical analyses," IEEE Access, vol. 8, pp. 4383-4396, December 2019. 